\title{
BMJ Open Patient-initiated questions: How can doctors encourage them and improve the consultation process? A qualitative study
}

\author{
G M Murtagh, ${ }^{1}$ L Furber, ${ }^{2}$ A L Thomas ${ }^{3}$
}

To cite: Murtagh GM, Furber L, Thomas AL. Patient-initiated questions: How can doctors encourage them and improve the consultation process? A qualitative study. BMJ Open 2013;3:e003112.

doi:10.1136/bmjopen-2013003112

- Prepublication history and additional material for this paper is available online. To view these files please visit the journal online (http://dx.doi.org/10.1136/ bmjopen-2013-003112).

Received 23 April 2013 Revised 10 July 2013 Accepted 11 July 2013

For numbered affiliations see end of article.

Correspondence to Dr G M Murtagh; g.murtagh@imperial.ac.uk

\begin{abstract}
Objective: To investigate the circumstances under which patients initiate direct questions in oncology consultations.

Design: Conversation analysis of 47 consultations between oncologists and patients with cancer.
\end{abstract}

Setting: An oncology clinic at a teaching hospital in the East Midlands.

Participants: 16 Oncologists and 67 cancer patients. Outcome measure: Patient initiated direct questions. Results: On the whole patients' direct questions are designed to seek specific information regarding, the cancer itself, treatment options or their experience of symptoms. When patients do ask direct questions they typically follow the announcement of test results where some reference to the details of those results, is provided. More specifically, there seems to be a relation between showing the patient their scan/X-ray results, patient involvement and patient-initiated direct questions. Higher levels of patient-initiated direct questions were clustered around occasions where doctors provided information and explanations of test results (12 consultations) sometimes with direct reference to scan or X-ray results (7 consultations).

Conclusions: This study highlights the importance of careful explanation of diagnostic evidence as a factor contributing to increased patient involvement. More specifically, the findings suggest that, when appropriate, invoking diagnostic evidence (eg, scan or $X$-ray results) is an effective way of increasing levels of patient question asking. Doctors need to be able to encourage patient question asking to ensure that patients have at their disposal an important means through which they can determine their information needs. Although these results come from a study of oncology consultations, the findings may be transferable to other clinical contexts.

\section{INTRODUCTION}

One of the main problems faced by oncologists in the consultation is the difficulty in accurately gauging the patient's information needs. ${ }^{1}{ }^{2}$ Studies have examined patient

\section{ARTICLE SUMMARY}

Strengths and limitations of this study

- The strength of the study is that it demonstrates a practical way for doctors to encourage patient involvement.

- Another strength is that it targets actual instances of question asking behaviour in relation to other situational variables in the consultation.

- The study is limited by its sole reliance on audio recordings of consultations. Consequently, other aspects of social interaction, for example, eye contact, bodily comportment, etc which can also have a significant influence on the content of the consultation, have not been included.

preferences for information provision and involvement ${ }^{1}{ }^{3-5}$ and have shown, among other things, that patients do want specific information concerning their illness. ${ }^{4}{ }^{5}$ However, patients' information needs are not static and there can be significant variations between patients in terms of their preferences for the timing, content and detail of information they require. Such variations may change during the course of an illness and even during the course of a single consultation depending on the type of information a patient receives. ${ }^{1}$ Consequently, patients' attempts to elicit information from doctors also varies. These contingencies indicate a real need to understand more about the conditions under which patients actively seek information and, more specifically, the kinds of communication behaviours patients use to seek information. ${ }^{6}$ Among these behaviours, question asking is key, as it provides the patient with a simple mechanism for information seeking. ${ }^{7}$ When patients ask questions it allows them to shape their own levels of involvement ${ }^{8}$ and handle the 
contingencies of information exchange. ${ }^{9}$ In addition, patient question asking has been linked to improved information provision. ${ }^{8}$ Moreover, patients who receive an answer to their question demonstrate better psychological adjustment following the consultation than those who ask questions but do not receive a response. ${ }^{11} 12$

How and when do patients ask questions? In the context of cancer care, research shows that direct questions (alongside indirect cues) occur most often during the treatment phase of the consultation ${ }^{13}$; that companions who accompany patients, tend to ask more questions than patients, particularly in relation to treatment and diagnsosis ${ }^{14}$; that ethnic and racial differences between patients can reflect differences in levels of question asking and direct question asking ${ }^{15}$; that question prompt lists can encourage patient question asking, particularly in relation to prognosis and diagnosis. ${ }^{16} 17$

These studies provide valuable direction in understanding some of the factors behind patient question asking in cancer care. However, the extent to which patients initiate information seeking, in the first place, is often contingent on the doctor's communicative style. The import of this lies in the fact that when patients seek information (eg, through asking questions), doctors typically respond in more informative and accommodative ways, ${ }^{18}$ partly because patient questions are one of the ways in which patients establish their information needs. The purpose of this study was to capture some of the interactional and situational variables that occur alongside patient initiated questions, to establish how and when patients are more inclined to initiate direct questions.

\section{METHODS}

The study was conducted in a large UK Cancer Centre. LF recruited patients (with different types of cancers) attending the oncology department $(n=77)$ as well as a mix of oncology consultants and specialist registrars. Newly diagnosed and follow-up patients were recruited to ensure maximum variability in our sample group. Following each consultation patients were invited to complete a satisfaction questionnaire and interviews were conducted with the patients by LF shortly after their consultation. Each of the interviews was analysed to identify common themes across the data until saturation was reached. Patient consent was obtained before their consultation was recorded and before collecting questionnaire data. This article reports on the recordings of the consultation data only.

We audio recorded 47 consultations which were then transcribed and analysed using conversation analysis, ${ }^{19}$ a method of analysis which details characteristics of speech exchange including pauses, pace and intonation and so on (the transcription symbols used to indicate these characteristics are provided below). In each

\section{Transcription symbols}

$\circ \circ$

(.)

(1.0), (0.5) indicates silence in seconds and tenths of seconds

[Okay

[Yes

Talk marked by the degree sound indicates words that are softly spoken

A full stop in brackets indicates a micro pause

overlap in speech between two different speakers

$=\quad$ Talk marked with the equals sign at the end of one line and the beginning of another indicates no pause between the end of one utterance and the start of another

consultation we identified the number of patientinitiated questions which arose. We then examined the location of these questions which allowed us to identify clusters around diagnostic news delivery. We also noted, however, that in other consultations patient-initiated questions were minimal or absent on occasions of diagnostic news discussion. This led us to question whether there was a relation between the doctor's communication behaviour and the patient's response when doctor and patient talked about test results. Using this as our focal point we identified 30 of the 47 consultations where the relation between style of diagnostic news delivery (elaborate/restricted) and patient response/involvement (patient initiated questions/no patient initiated questions) was most clear. From this sample of 30 we selected eight examples (discussed below) which in our view provided the strongest indication of how the style of delivery of news/results can influence patient involvement/questions. This sample of eight also allows us to demonstrate most clearly the contrast between the two different styles of delivery, restricted and elaborate. Transcription and analysis was conducted by GM. Subsequent analyses were conducted by GM, AT and LF. Any disagreements regarding interpretation of the data were resolved through discussion and by revisiting the data. Inclusion criteria: patients over the age of 18, having been diagnosed with cancer, aware of their diagnosis and willing to participate in the study. Exclusion criteria: any patient unable to consent for themselves, patients with a cognitive impairment and patients who do not speak fluent English.

\section{ANALYSIS}

For the purposes of analysis we defined a direct question as that which is initiated solely by the patient, without a verbal prompt ('Do you have any questions?') from the doctor and which targets a specific topic. The consultations were examined with a view to identifying some of the systematic and recurrent properties of the delivery and receipt of test results. More specifically, we examined how styles of news delivery shape patients' responses, in particular their levels of question asking. 


\section{RESULTS}

Our focused sample of 30 consultations in total came to $451.30 \mathrm{~min}$, just over $7.5 \mathrm{~h}$ of consultation time with the average length of the consultation at $15.04 \mathrm{~min}$. In 7 of the 30 consultations (just under $60 \mathrm{~min}$ of consultation time) patients did not ask any questions. In the remaining 23 consultations there were 76 instances of patients asking questions (average 2.5 direct patient questions per consultation). However, patients' questions arose in different ways. For example, in 5 of those 23 consultations $(22 \%)$ patients' questions came at the end (within 3-4 min of the end of the consultation) again following a prompt from the doctor. In 6 of the 23 consultations (26\%) there is evidence of indirect or embedded questions arising at different junctures of the consultation following a prompt from the doctor. In 12 of the 23 consultations (52\%), patient-initiated direct questions occur specifically in relation to discussion of test results. In 7 of these 12 consultations (58\%) patient-initiated question asking occurs following a careful explanation of test results and diagnostic evidence, for example, the doctor's use of scan or X-rays. In only two consultations did the patient decline to ask a question following an invitation to do so from the doctor.

We noted several variations in the manner in which doctors deliver test results. Our main finding, however, is that patients are more inclined to initiate direct questions when doctors deliver results with direct reference to the diagnostic evidence, for example, X-rays or scans. To exemplify this we identified two types of information delivery each resulting in different types of patient response; the most marked difference being levels of patient initiated question asking. In delivery type 1 , test results were delivered in a very general way without elaboration (restricted delivery-eg, 'your scan results are fine'). With this type of delivery patient-initiated questions were absent or minimal. In delivery type 2 (Elaborate delivery-'your scan shows that...') the doctor elaborated or explained the test results sometimes invoking the scan or the X-ray to do so. This type of delivery typically positively influenced levels of patient involvement in the consultation and prompted more patient-initiated direct questions and consequently more information provision from the doctor.

\section{Restricted delivery}

The sequences in boxes 1-4 show the announcement of diagnostic results depicting the type 1 delivery. In each case the results are delivered in a general, non-specific manner characterised by a general clinical assessment, 'fine', 'normal' or 'no change'. In each case such delivery result in a minimal response from the patient. It seems that the general delivery in type 1 projects a paternalistic approach where the doctor presents his/her interpretation of the results as the authoritative one, without any specific reference to further details of the findings. ${ }^{20}$ Consequently, the patient is invited to accept the diagnostic judgement of the doctor and the general,

\section{Box 10 Patient-initiated questions}

1. Doctor: The CT scan result is here (0.5) and that was

2. basically normal erm nothing to suggest any new no new

3. glands you have got some changes on your erm (4.0) lungs

4. from(.) previous radiotherapy (0.5)uhm (1.5) so that's your

5. CT scan and I'm just trying to find the (0.5) lung function

6. tests(.) when did you have those done

7. Patient: (2.0) had them done

8. Husband: Two weeks ago

\section{Box 20 Patient-initiated questions}

1. Doctor: Okay (.) um (0.5) scan result was fine

2. Patient: Good

3. Doctor: Good okay an everything's stable on the in the

4. bones

5. Patient: Right

\section{Box 30 Patient-initiated questions}

1. Doctor: Your scan shows everything is the same

2. Patient: Good

3. Doctor: So that's very good

\section{Box 4 Patient-initiated question}

1. Doctor: And you've had an echocardiogram of your heart

2. an that's all fine

3. Patient: Is it [okay?

4. Doctor: [you had that done on?

5. Patient: Last Friday

6. Doctor: Last Friday that's all fine (.) no problems so

7. that's good news could I er examine you

non-specific explanation of the results is reflected in the general, non-specific response provided by the patient. ${ }^{21}$ In almost a third of the sample, apart from the sequence in box 4, the patient does not question or inquire further into the results following the type 1 delivery. In box 4 the patient does ask a question, but again this is presented in a general form 'is it okay?' again reflecting the general way in which the result of the echocardiogram is presented. However, later on following a physical examination the patient targets back on this assessment, after a physical examination, following a prompt from the doctor.

1. Doctor: Is there anything you wanted to ask at all?

2. Patient: I did want to ask about my heart function

3. Doctor: Ya sure.

4. Patient: I know you said the echocardiograms are okay

5. Doctor: Yes

6. Patient: but has it (.) erm deteriorated at all[through

7. Doctor: [no no 


\section{Elaborate delivery}

\section{Box 51 Patient-initiated question}

1. Doctor: The head scan, the CT and the MRI show that there

2. is something in the bones but what is unusual is that it

3. seems to be more on the right hand side than the left and I

4. think it was the left eye you were having problems with?

5. Patient: (0.5) Don't they cross?

6. Doctor: Well sometimes if it's more of a visual problem

7. they do cross yeah

In this sequence there are two distinctive features which appear to shape the patient's response. First, the doctor delivers the findings from the scan and produces an expression of uncertainty regarding which eye the patient was having problems with. Second, there is a half second pause following the delivery which not only provides the patient with the opportunity to respond but also scope to negotiate the nature of that response.

\section{Box 65 Patient-initiated questions}

1. Doctor: Your scan shows that things are very much the

2. same, maybe slightly bigger but literally by $4 \mathrm{~mm}$ both in

3. the chest and in the bowel

4. Patient: Is it possible for me to see the scan?

5. Doctor: Yes, these are your lungs, that's your heart

6. Patient: Where's the tumor?

7. Doctor: That's it

8. Patient: It's there? So when I saw it previously it was

9. about that size?

10. Doctor: It's only a couple of centimetres most

11. Patient: As small as that? In fact it's smaller

12. than when I first came about walnut size

13. Doctor: It doesn't really say how big it was

14. initially

15. Patient: So it would be about like that wouldn't it?

16. Doctor: Yeah

17. Patient: It was on the lymph gland, is that the lymph

18. gland?

19. Doctor: No that's your bowel that's the tumor and

20. that's your bowel there and that's your aneurism

21. they've measured it for you $55 \mathrm{~mm}$

In this next sequence, the results are delivered and carefully explicated with the inclusion of numerical data specifying the size of the cancerous growth (lines 1-3). This provides the patient with a precise frame of reference regarding the cancer. Interestingly in this case the patient, in response, asks to see the scan (line 4). The doctor then identifies the patient's lungs and heart providing the relevance for the proximal distance of the tumour from the lungs and heart. The patient (line 6) then asks about the location of the tumour, its size (lines $8-9,11$ ) and finally asks about the lymph gland (lines 17 and 18).

In the sequence in box 7 the delivery of diagnostic news starts out almost as a type 1 delivery ('Your scan

\section{Box 73 Patient-initiated questions}

1. Doctor: The scan is very much the same erm there hasn't

2. been um any obvious problems there is quite a lot of

3. fibrosis still but that's to be expected so fibrosis is

4. healing and scarring

5. Patient: Where's that?

6. Doctor: Umm both in the air in the central areas you know

7. where all the problems originally were with the

8. swallowing so in the central area and in the tummy um (2.0)

9. let me tell you exactly

10. Patient: Was that there before?

11. Doctor: They've said there's an increase in the volum

12. of that fibrosis

13. Patient: So basically that's scar tissue, is that what

14. you're saying?

shows that things are very much the same') but then goes on to point out that fibrosis is still present which is 'to be expected'. The doctor then explains the term fibrosis and the patient responds (line 5) by asking where it is. When the doctor explains the location of the fibrosis, the patient asks if it was present before. The doctor then refers to the report regarding the increase in fibrosis and the patient (line 13) then presents a gloss of the meaning of the news which is posed as a question.

\section{Box 85 Patient-initiated questions}

1. Doctor: So they've reported it as stable disease basically

2. nothing new to find there are some lymph nodes in your

3. pelvis but there's nothing different from that

4. Patient: Just where exactly?

5. Doctor: Did you want to look at your scan you [can

6. Patient:

7. able to tell from that?

8. Doctor: Well we can look at it together

9. Patient: Yeah

10. Doctor: (3.0) So this is your pelvis

11. Patient: Right

12. Doctor: This is your right hip and that is your left hip=

13. Patient: $=\mathrm{Mhm}$

14. Doctor: And then you've got some lymph nodes that are

15. predominantly on the on the right hand side

16. Patient: Yes right so the other side is what they

17. should look like is it?

18. Doctor: Yeah you've got some tiny lymph nodes there

19. they're normally a centimeter and a half is as big as

20. you'd expect them to be normally

21. Patient: Right

22. Doctor: You have got some higher up as well

23. Patient: So that's more into the tummy?

24. Doctor: Yeah

25. Patient: Dya think it is possible that thee enlarged

26. (.) lymph nodes could be (0.5) pressing on a ne::rve

27. [or

28. Doctor: [Sometimes they can do ya ya 
The diagnostic news delivery specifies the fact that there 'are some lymph nodes' (box 8). In response the patient asks 'where exactly' (line 4). The doctor then asks the patient if they would like to look at their scan. Interestingly the patient's next question ('Will I be able to tell from that'?) at lines 6-7 manifests the knowledge-competence gap between doctors and patients. This may partly account for why doctors do not always invite patients to look at scan/X-ray results and why patients do not always ask to see them when they are available. The doctor's response ('we can look at it together') bridges this gap by inviting the patient to examine the scan jointly allowing the doctor to identify, for the patient, key anatomical structures while also allowing the patient to inquire further.

The sequences in boxes 5-8 show the announcement of diagnostic results depicting the type 2 delivery. In these sequences the results are delivered alongside a clinical assessment which either includes a numerical reading or further explication of the findings. When results are delivered in this way, patients tend to engage with the doctor. An important consequence of this is higher levels of patient involvement including more patient initiated questions.

\section{DISCUSSION}

In the main, consultations covered topics such as treatment, the progression of the cancer itself and the symptoms experienced by the patient. Not all topics were addressed in every consultation and doctors varied in how they dealt with each topic. We found that generally patients' actual levels of involvement in the consultation were relatively low and patients varied in how active they were in seeking information. We also found that, on the whole, patients seemed disinclined to ask questions or show communication behaviours designed to elicit information. This finding is consistent with much earlier research into this topic. ${ }^{6} 17$

In relation to discussions of test results between doctor and patient, the data appear to indicate that there may be a connection between the manner in which the results are delivered and the occurrence of patient-initiated direct questions. That is to say, the plainer announcement of diagnostic results ('your scan is fine'-type 1), which does not include sharing the diagnostic evidence projects a more paternalistic approach implicit in which, the patient is expected to accept the diagnostic judgement of the doctor. It is also characterised by a general explanation of the results which is typically reflected in the general response provided by the patient. ${ }^{21}$ In contrast, the type 2 delivery is much more accommodative of patient input. Moreover, the invocation of the scan or the X-ray, where appropriate, appears to be significant in encouraging patient involvement and consequently patient-initiated direct questions. Incorporating and explaining the evidence appears to be interpreted by patients as an opportunity to contribute to the consultation and establish their information needs in an environment within which the patient's queries/opinions are welcomed. Unless the patient has specifically requested not to see scans or $\mathrm{X}$-rays, this would appear to be an effective way of encouraging patient involvement generally and increasing levels of patient question asking. Consequently, patients are then able to establish and satisfy their information needs in a timely and effective manner.

In cases in which patients did ask more questions, there was no significant increase in consultation length and no patient refused the offer of looking at diagnostic results. The examples presented above were carefully selected because they display the most marked variation in consultation style highlighting clear contrasts between the two types of delivery.

Various types of intervention have been used in cancer care to help facilitate patient involvement. For example, question prompt lists have been used quite widely, but their actual implementation in consultations is not always straightforward and their rates of success do vary. ${ }^{15}$ Moreover, we found in our study, that even when patients entered the consultation with question lists, they often left the consultation without having asked the questions they came prepared for.

The finding that when the doctor elaborates or explicates findings from the evidence, this can increase levels of patient involvement has been identified previously in a study of primary care consultations. ${ }^{22}$ Clearly, there are important differences between an oncology consultation and a primary care consultation. However, in relation to consulting behaviours, in both settings there appear to be striking similarities. That is to say, careful explication of diagnostic findings can encourage patient involvement such as, in this case, question asking, which in turn can enable patients to establish their information needs. Further research in this demands a closer investigation of what Frankel ${ }^{9}$ has described as the "presuppositional grounds upon which the communication situation itself rests'. This would then allow us to identify other consulting behaviours doctors can utilise to encourage patient involvement in general.

There may be various reasons why patients were disinclined to ask questions following the "your scan result is fine' type of announcement. The minimal responses identified may not actually be conditioned solely by the type of announcement of test results but may also be a consequence of patient preference or information needs at that particular moment. As noted, in type 1, box 4 the patient targets back on a general assessment of the echocardiogram as 'okay'. This clearly merits further empirical investigation.

\section{CONCLUSION}

Currently there is good research evidence indicating that patient-initiated question asking should be encouraged. Doctors need to be able to encourage patient question asking to ensure that patients have at their disposal an important means through which they can determine and 
express their information needs. This study confirms the findings from previous studies showing that levels of patient initiated questions in Oncology are relatively low. Our study also suggests that patient-initiated question asking can be encouraged through timely and deliberate information giving which incorporates an explanation and display of test results. The findings at this stage are only suggestive. Studies which involve closer examination of the actual interactional episodes between doctors and patients are required to provide a deeper understanding of patient-initiated questions and the situational variables which may influence them.

Author affiliations

${ }^{1}$ Division of Surgery, Department of Surgery and Cancer, St Mary's Campus, Imperial College London, London, UK

${ }^{2}$ Department of Cancer Studies and Molecular Medicine, Robert Knighton Clinical Sciences Building, University of Leicester, Leicester Royal Infirmary, Leicester, UK

${ }^{3}$ Department of Cancer Studies and Molecular Medicine, Leicester Royal Infirmary, Leicester, UK

Contributors PI and AT led the study design with contributions from GM and LF. GM and LF were involved in data collection and analysis with contributions from AT. GM was involved in preparing the first draft of the manuscript with contributions from AF and ALT towards data interpretation and reviewing of the manuscript.

Funding This article presents independent research funded by the National Institute for Health Research (NIHR) under its Research for Patient Benefit (RfPB) Programme (Grant Reference Number PB-PG-0807-14122).

Competing interests None.

Ethics approval The study received ethical approval from the Nottingham research ethics committee 2 ID: 09/H0408/34.

Provenance and peer review Not commissioned; externally peer reviewed.

Data sharing statement No additional data are available.

Open Access This is an Open Access article distributed in accordance with the Creative Commons Attribution Non Commercial (CC BY-NC 3.0) license, which permits others to distribute, remix, adapt, build upon this work noncommercially, and license their derivative works on different terms, provided the original work is properly cited and the use is non-commercial. See: http:// creativecommons.org/licenses/by-nc/3.0/

\section{REFERENCES}

1. Butow PN, Maclean M, Dunn SM, et al. The dynamics of change: cancer patients' preferences for information, involvement and support. Ann Oncol 1997;8:857-63.
2. Gattellari M, Butow PN, Tattersall MHN. Sharing decisions in cancer care. Soc Sci Med 2001;52:1865-78.

3. Leydon GM, Boulton M, Moynihan C, et al. Cancer patients information needs and information seeking behaviour: in-depth interview study. BMJ 2000;320:909-13.

4. Jenkins V, Fallowfield L, Saul J. Information needs of patients with cancer: results from a large study in UK cancer centres. Br J Cancer 2001;84:48-51.

5. Cox A, Jenkins V, Catt S, et al. Information needs and experiences: an audit of UK cancer patients. Eur J Oncol Nurs 2006;10:263-72.

6. Cegala D, Marinelli T, Post D. The effect of patient communication skills training on compliance. Arch Fam Med 2000;9:957-64.

7. Beisecker AE, Beisecker TD. Patient information seeking behaviours when communicating with doctors. Med Care 1990;28:19-28.

8. Street RL, Millay B. Analysing patient participation in medical encounters. Health Commun 2001;13:61-73.

9. Frankel R. Talking in interviews: a dispreference for patient initiated questions in physician-patient encounters. In: Psathas G. ed. Interaction competence. Lanham, MD: University Press of America, 1990:231-61.

10. Cegala D, Street RL, Clinch RC. The impact of patient participation on physicians' information provision during a primary care medical interview. Health Commun, 2007;21:177-85.

11. Butow PN, Dunn SM, Tattersall MHN, et al. Computer-based interaction analysis of the cancer consultation. Br J Cancer 1995;71:1115-21.

12. Street RL. Information giving in medical consultations: the influence of patients' communicative styles and personal characteristics. Soc Sci Med 1991;32:541-8.

13. Butow PN, Brown RF, Cogar S, et al. Oncologists reactions to cancer patients' verbal cues. Psychooncology 2002;11:47-58.

14. Eggly S, Penner LA, Greene M, et al. Information seeking during "bad news" oncology interactions: question asking by patients and their companions. Soc Sci Med 2006;63:2974-85.

15. Eggly S, Harper FWK, Penner LA, et al. Variation in question asking during cancer clinical interactions: a potential source of disparities in access to information. Patient Educ Couns 2011;82:63-8.

16. Brown R, Butow PN, Boyer MJ, et al. Promoting patient participation in the cancer consultation: evaluation of a prompt sheet and coaching in question asking. Br J Cancer 1999;80:242-8.

17. Dimoska A, Tattersall MHN, Butow PN, et al. Can a "prompt list" empower cancer patients to ask relevant questions. Cancer 2008:113:225-37.

18. Street RL, Gordon HS, Ward MM, et al. Patient participation in medical consultations: why some patients are more involved than others. Med Care 2005;43:960-9.

19. Heritage J, Maynard D. Communication in medical care: interaction between primary care physicians and patients. Cambridge: Cambridge University Press, 2006

20. Pomerantz A, Sean Rintel E. Practices for reporting and responding to test results during medical consultations: enacting the roles of paternalism and independent expertise. Discourse Stud 2004;6:9-26.

21. Collins S, Drew P, Watt I, et al. 'Unilateral' and 'bilateral' practitioner approaches in decision-making about treatment. Soc Sci Med 2005;61:2611-27.

22. Perakyla A. Communicating and responding to diagnosis. In: Heritage J, Maynard D. eds. Communication in medical car: Interaction between primary care physicians and patients. Cambridge, UK: Cambridge University Press. 2006:214-47. 